\title{
The Infrared Imaging Spectrograph (IRIS) for TMT: The Science Case
}

\author{
Elizabeth J. Barton ${ }^{a}$, James E. Larkin ${ }^{b}$, Anna M. Moore ${ }^{c}$, Shelley A. Wright ${ }^{d}$, David \\ Crampton $^{e}$, Luc Simard ${ }^{e}$, Bruce Macintosh ${ }^{f}$, Patrick Côtée ${ }^{e}$ Aaron J. Barth ${ }^{a}$, Andrea M. \\ Ghez $^{b}$, Jessica R. Lu ${ }^{g}$, T. J. Davidge ${ }^{e}$,David R. Law ${ }^{b}$, and the IRIS Science Team \\ ${ }^{a}$ Center for Cosmology, University of California, Irvine, USA; \\ ${ }^{b}$ Division of Astronomy \& Astrophysics, University of California, Los Angeles, USA; \\ ${ }^{c}$ Caltech Optical Observatories, California Institute of Technology, USA; \\ ${ }^{d}$ Department of Astronomy, University of California, Berkeley, USA; \\ ${ }^{e}$ Herzberg Institute of Astrophysics, National Research Council Canada

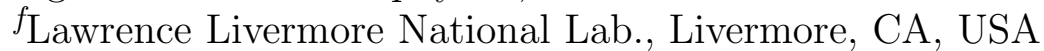 \\ ${ }^{g}$ California Institute of Technology, Pasadena, CA, USA
}

\begin{abstract}
The InfraRed Imaging Spectrograph (IRIS) is a first-light instrument being designed for the Thirty Meter Telescope (TMT). IRIS is a combination of an imager that will cover a $16^{\prime \prime} 4$ field of view at the diffraction limit of TMT (4 mas sampling), and an integral field unit spectrograph that will sample objects at 4-50 mas scales. IRIS will open up new areas of observational parameter space, allowing major progress in diverse fields of astronomy. We present the science case and resulting requirements for the performance of IRIS. Ultimately, the spectrograph will enable very well-resolved and sensitive studies of the kinematics and internal chemical abundances of high-redshift galaxies, shedding light on many scenarios for the evolution of galaxies at early times. With unprecedented imaging and spectroscopy of exoplanets, IRIS will allow detailed exploration of a range of planetary systems that are inaccessible with current technology. By revealing details about resolved stellar populations in nearby galaxies, it will directly probe the formation of systems like our own Milky Way. Because it will be possible to directly characterize the stellar initial mass function in many environments and in galaxies outside of the the Milky Way, IRIS will enable a greater understanding of whether stars form differently in diverse conditions. IRIS will reveal detailed kinematics in the centers of low-mass galaxies, allowing a test of black hole formation scenarios. Finally, it will revolutionize the characterization of reionization and the first galaxies to form in the universe.
\end{abstract}

Keywords: Integral Field Spectrograph, Thirty Meter Telescope, Infrared Instrumentation, Science Case

Further author information: (Send correspondence to E.J.B.)

E.J.B.: E-mail: ebarton@uci.edu.edu, Telephone: 19498247178

J.E.L.: E-mail: larkin@astro.ucla.edu, Telephone: 13108259470

A.M.M.: E-mail: amoore@astro.caltech.edu, Telephone: 16263958918

S.A.W.: E-mail: saw@astro.berkeley.edu, Telephone: 13109807060

D.C.: E-mail: David.Crampton@nrc-cnrc.gc.ca, Telephone: 12503630010

L.S.: E-mail: Luc.Simard@nrc-cnrc.gc.ca, Telephone: 12503638315

B.M.: E-mail: macintosh1@llnl.gov, Telephone: 19254238129

P.C.: E-mail: Patrick.Cote@nrc-cnrc.gc.ca, Telephone: 12503638133

A.J.B.: E-mail: barth@uci.edu, Telephone: 19498243013

A.M.G.: E-mail: ghez@astro.ucla.edu, Telephone: 13102060420

J.R.L.: E-mail: jlu@astro.caltech.edu, Telephone 16263953693

T.J.D.: E-mail: Tim.Davidge@nrc-cnrc.gc.ca, Telephone: 12503630047

D.R.L.: E-mail: drlaw@astro.ucla.edu, Telephone: 13107949466

Ground-based and Airborne Instrumentation for Astronomy III, edited by lan S. McLean,

Suzanne K. Ramsay, Hideki Takami, Proc. of SPIE Vol. 7735, 77355M · @ 2010

SPIE · CCC code: $0277-786 X / 10 / \$ 18 \cdot$ doi: $10.1117 / 12.856521$

Proc. of SPIE Vol. 7735 77355M-1 


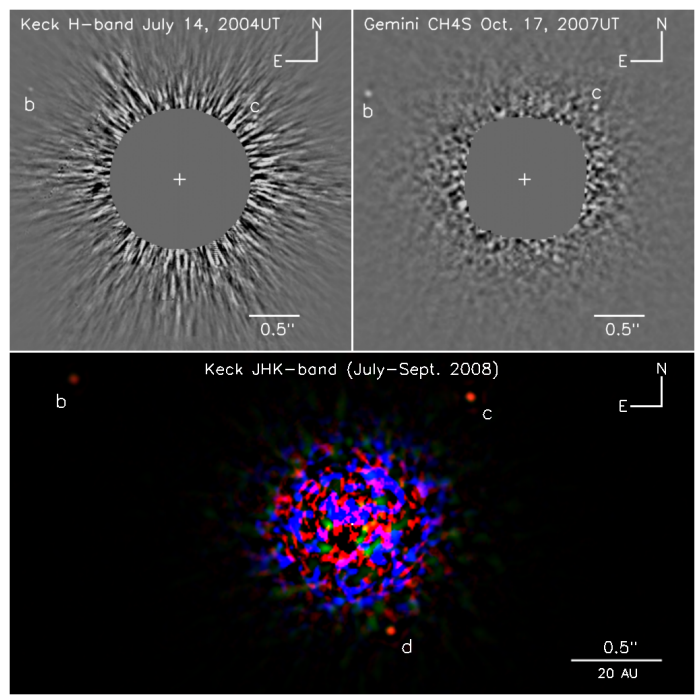

Figure 1. HR8799bcd discovery images from, ${ }^{5}$ including (Upper left) a Keck image, (Upper right) a Gemini image, and (Bottom) a color image from the Keck data showing three planets around the parent star. The combination of TMT and IRIS will excel at detecting relatively low-contrast planets that are as close as $\sim 00^{\prime \prime} 1$ to their parent stars.

\section{INTRODUCTION}

As a premier first-light instrument for the Thirty Meter Telescope, IRIS ${ }^{1}$ will open huge windows of astronomical discovery space ${ }^{2}$ paving the way for a new era of exploration with extremely large telescopes. Operational in the near-infrared (0.8-2.4 $\mu \mathrm{m})$, IRIS will offer diffraction-limited imaging and integral-field spectroscopy behind the TMT facility Narrow-Field Infrared Adaptive Optics system (NFIRAOS). ${ }^{3}$ With 4 spatial scales for spectroscopic sampling $(4,9,25$, and 50 mas), its versatility will allow the efficient study of both unresolved and diffuse objects. Here, we describe a subsample of its many breakthrough scientific capabilities.

\section{IMAGING AND SPECTROSCOPY OF EXOPLANETS}

The direct imaging detection of extrasolar planets, and their characterization via spectroscopy, remain frontier topics in astrophysical research. In the exciting hunt for an understanding of planets outside of our own Solar system, IRIS on TMT will combine extreme sensitivity and spectroscopic resolution in the range of $R \sim 1000-$ 8000 to complement special purpose planet hunting instrumentation. IRIS will also bridge the gap until highcontrast instruments such as the Planet Formation Imager ${ }^{4}$ come online for TMT.

IRIS will excel at higher-resolution $(R \geq 200-1000)$ spectroscopic study of known self-luminous extrasolar planets at large separations, where scattered light is not the limiting factor. Examples include the companions of $\mathrm{HR} 8799^{5}$ shown in Fig 1. Intense spectroscopic study of these and other planetary companions will yield unprecedented information about their gravity, composition, and atmospheres. Thus, this science case drives requirements on the sensitivity of IRIS, as well as the point-spread function and the dynamic range.

Also at the forefront of extrasolar planetary research, the angular resolution afforded by the thirty meter primary aperture of TMT will allow IRIS to excel over other planned instrumentation at discovering bright planets that are very close to their parent stars, $\leq 0^{\prime \prime} 1$, which corresponds to 10-15 AU in star-forming regions and $\sim 5 \mathrm{AU}$ in young stellar associations. These objects would include forming protoplanets in, e.g., Taurus and Ophiuchus, which are often too dim and red for higher-contrast AO systems like the Gemini Planet Imager. ${ }^{6}$

\section{BLACK HOLES}

The mysterious black holes predicted by Einstein's theory of General Relativity (GR) provide remarkable laboratories for the study of physics in extreme environments. Progress in this field drives instrument requirements on 

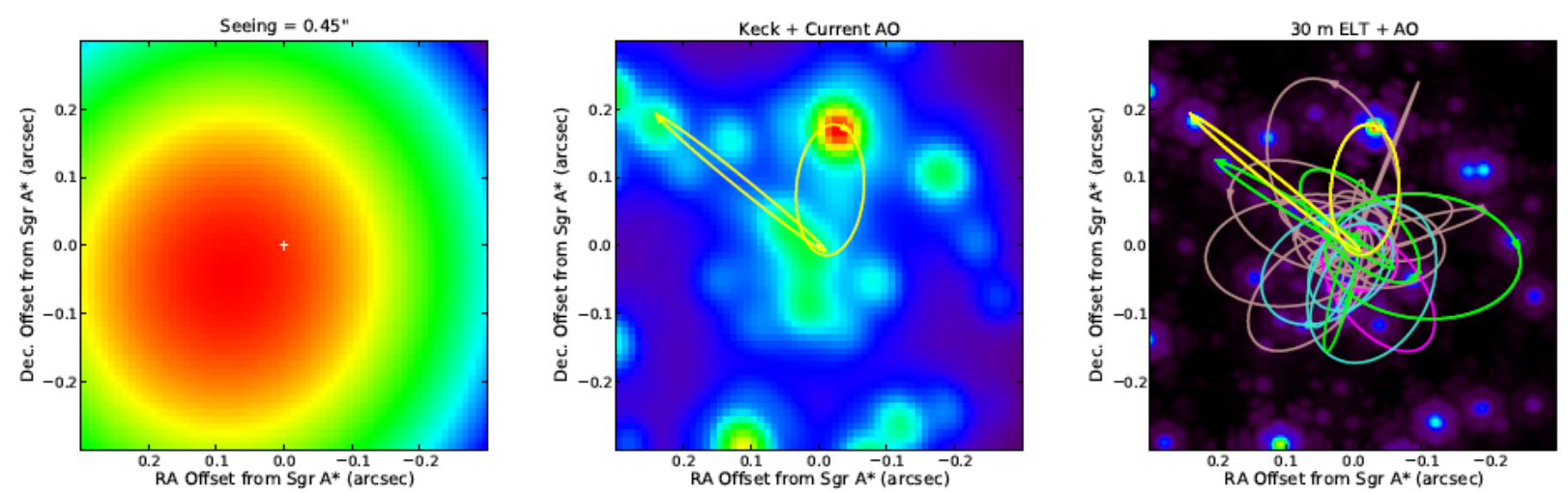

Figure 2.

Probing the Galactic Center with TMT, courtesy of A. Ghez, N. Weinberg, M. Morris, and J. Lu. The figure shows a seeing-limited observation of the $0^{\prime \prime} 6$ region of the Galactic Center (Left), an image with the current Keck adaptive optics capabilities (Middle), and a simulation of the Galactic Center as observed by TMT (Right). The IRIS instrument on TMT will reveal the orbits of many fainter and closer probes of the Galactic Center potential well..

the combination of angular resolution, which allows gravitational tests much closer to the black holes themselves and will make use of the diffraction-limited capabilities of the instrument, and sensitivity, which is required to probe a larger and/or more abundant population of "test masses" in order to understand the black holes' effects. In the case of the Galactic Center, the need to trace the orbits of stars also drives the strict astrometric requirements on IRIS (50 $\mu$ as). Thus IRIS on TMT will be an ideal instrument with which to study these elusive bodies.

\subsection{Fundamental physics from the black hole at the center of the Galaxy}

For the first time, IRIS will extend our knowledge of the spacetime topology around a supermassive black hole to well within $~ 1000$ Schwarzchild radii (see Fig. 2). The immediate surroundings of the black hole at the center of our Galaxy will allow fundamental tests of "medium field" relativity, including the astrometric signal of prograde GR precession, ${ }^{7}$ the influence on radial velocity measurements from the special relativistic transverse Doppler shift, and the general relativistic gravitational redshift. ${ }^{8}$

Because the detailed orbits of stars in the vicinity of the central black hole will be affected by the presence or absence of dark matter, the orbits of stars within $0.01 \mathrm{pc}$ of the Galactic Center will significantly constrain the dark matter models on which galaxy formation is built. With a decade of astrometric measurements using IRIS on TMT, this orbital influence would be readily detectable, standing ready to confirm theoretical predictions of the amount of Galactic dark matter within the very center of the Galaxy. Finally, at its astrometric requirement ( 50-100 $\mu$ as), IRIS on TMT will provide a very precise measurement of the distance to the Galactic Center, $\mathrm{R}_{0}$. A measure within $1 \%$ would enable an accurate determination of the size and shape of the Milky Way's dark matter halo on $100-k p c$ scales. ${ }^{9}$

\subsection{Black holes in other galaxies}

Since the discovery that black holes reside in the centers of most, if not all, massive galaxies, their role in galaxy formation and evolution has been at the forefront of astronomical research. Direct measurements of their masses require both spatially well-resolved spectroscopy at high spectral resolution $(R \sim 4000-8000)$. For the first time, the sensitivity afforded by IRIS on TMT will allow these measurements to extend to more distant galaxies and to fainter, lower mass galaxies. Thus, it will extend our knowledge of both the high-mass and low-mass ends of the relationship between black hole masses and bulge velocity dispersion, $\sigma$ (Fig. 3), which is a fundamental probe of the relationship between black hole growth and galaxy formation. 

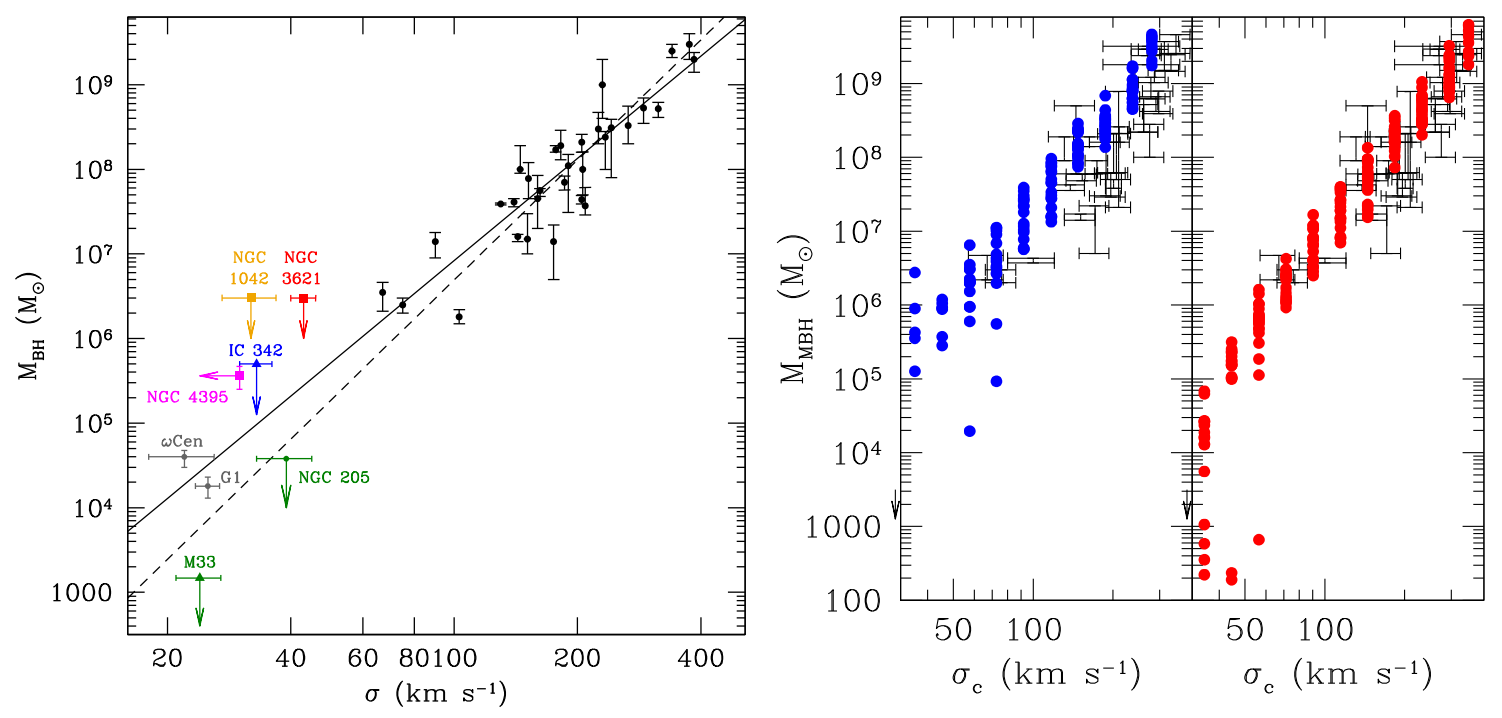

Figure 3.

(Left) The black hole mass - bulge velocity dispersion relation from Barth et al. (2009a), based on new work and a compilation from the literature. The relation demonstrates the deep connections between the formation of bulges and spheroids and the formation of central supermassive black holes in galaxies. (Right) Models of black hole masses from 10, using two formation scenarios adapted from 11. The model on the left has $10^{5}$ solar mass seed black holes, while the model on the right starts with 100-solar-mass black holes originating from Population III stars. IRIS will measure the masses of low-mass black holes in the nearby universe, distinguishing between these scenarios.

Low-mass black holes and galactic nuclei are of particular interest for black hole formation scenarios. Recent studies with the Hubble Space Telescope (HST) have shown that the vast majority of low-luminosity galaxies contain compact stellar nuclei (sometimes referred to as nuclear star clusters) whose properties are also connected to those of their hosts. It is not clear whether these low- and intermediate-luminosity galaxies contain supermassive black holes as well. From an observational perspective, detecting such black holes is extremely challenging because of their comparatively "modest" masses: i.e., for an intermediate-luminosity galaxy $\left(\mathrm{M}_{\mathrm{B}} \sim 17.0\right.$ magnitudes and $\sigma \sim 40 \mathrm{~km} \mathrm{~s}^{-1}$ ) the $\mathrm{M}_{\mathrm{BH}^{-}} \sigma$ scaling relation predicts $\mathrm{M}_{\mathrm{BH}} \sim 46,000-153,000$ solar masses, with the precise value depending on the choice of the $\mathrm{M}_{\mathrm{BH}^{-}} \sigma$ relation used for the extrapolation.

Characterizing the central mass concentrations in low- and intermediate-luminosity galaxies will be a top priority with 30m-class telescopes, and IRIS will be the instrument of choice for such studies with TMT. A key open question is the age and abundance distributions of the nuclei since, in principle, these provide information on the history of star formation and chemical enrichment on the smallest spatial scales in galaxies (with obvious implications for the fueling of the central black hole and AGN feedback). The observations are challenging, though, because of the small sizes of the nuclei $\left(0{ }^{\prime \prime} 05=4 \mathrm{pc}\right.$ at the distance of the Virgo Cluster, the nearest collection of suitable targets), the enhanced "sky" brightness due to the underlying galaxy, and the need for moderate or higher spectral resolutions $(R \geq 4000-8000)$. The results promise fundamental insights into black hole formation, however, because different formation scenarios yield very different predictions for the distribution of black hole masses at the low-mass end (see Fig. 3).

\section{RESOLVED STELLAR POPULATIONS IN THE LOCAL GROUP}

What are the chemical properties of bulge stars, and how do these vary with location in a bulge? What fraction of the stars in the inner regions of bulges originated from material that originated in the disk, and how far do these stars diffuse from their places of birth? How do the properties of stars in the Galactic bulge compare with those in other nearby spheroids? Answers to these questions can be found by investigating stars in the central regions of spheroids. Unfortunately, crowding makes it difficult to resolve individual stars in the central regions 
of even the closest external galaxies. As a result, an understanding of resolved stellar populations is a major driver on the image quality requirements for IRIS.

With the possible exception of space-based facilities, efforts to resolve individual stars in the main bodies of bulges are - by necessity - made in the near-infrared wavelength region. Not only is this the wavelength regime where ground-based telescopes deliver their best angular resolution, but spheroids tend to be comprised of stars with relatively high metallicities, and line blanketing can severely affect the brightnesses of evolved stars with metallicities that are with a tenth of solar. As a consequence, observations made at visible wavelength are biased against the detection of the most metal-rich giants, which will be fainter than their more metal-poor kin. While the visible wavelength range contains many well-calibrated features that have traditionally been used for abundance studies, the K-band also contains lines that can be used to measure the strengths of various atomic species. $^{12}$

Only the very brightest AGB stars can be resolved in the central 100 parsecs of Local Group galaxies with ground-based 4-8 meter telescopes. ${ }^{13-15}$ Because of the high angular resolution that will be delivered by TMT+NFIRAOS, IRIS will offer a unique capability for resolving individual stars in the central regions of M31, M32, and M33, and characterizing their stellar contents. Not only will the increase in angular resolution allow deeper observations than those conducted with 4 - 8 meter telescopes, but with integral field spectroscopic velocity measurements one can also identify individual stars in extremely crowded kinematically hot environments. ${ }^{16}$

\section{KINEMATICS AND METALLICITY VARIATIONS INSIDE HIGH-REDSHIFT GALAXIES}

A major breakthrough scientific application of IRIS+NFIRAOS will be the spatial dissection of galaxies during the peak epoch of galaxy formation in the range $z \sim 1-5$, the most active known period of star formation and AGN accretion in the history of the universe. Observations of these galaxies with the TMT will exploit both its light gathering power and its unique angular resolution at near-IR wavelengths. Large samples of galaxies throughout this redshift range are already known, and the current generation of 8-10 m telescopes has recently provided intriguing evidence for prevalent dynamically "hot" high-velocity dispersion systems that do not fit neatly into our current picture of galaxy formation. ${ }^{17}$ TMT and IRIS will allow observations of a much broader range of systems, and of systems at higher redshifts (see Fig. 4). ${ }^{2,18}$

Spatially resolved spectroscopy of emission lines with the light-gathering power and spatial resolution of TMT will allow differences in chemistry, kinematics, and physical conditions to be mapped as a function of spatial position within the galaxies. These measurements are required to go beyond our current knowledge of crude global properties, gaining a new level of understanding of the physics of galaxy formation.

IRIS, with its imaging and IFU capabilities, will provide crucial steps in a comprehensive survey of these systems. It will measure velocity widths and global rotation of galaxies, helping to distinguish kinematics associated with ongoing merging signatures from kinematics of rapidly star-forming but otherwise undisturbed galaxies. By measuring emission line ratios as a function of position within the galaxies, IRIS will uncover active galactic nuclei that might otherwise be hidden, and it will probe the spatial evolution of metallicity gradients within galaxies, which are necessarily closely tied to their formation mechanisms. ${ }^{19,20}$ The need to sample both the lumpy and the diffuse parts of high-redshift galaxies with maximal sensitivity is a significant driver on the IRIS IFU's selectable sampling scale, which includes both fine (4, 9 mas) and coarse (25, 50 mas) sampling.

\section{FIRST LIGHT AND REIONIZATION}

Sometime during the first 800 million years after the Big Bang $(z>7)$, the first tiny seeds of galaxies began to collapse and to form stars. Understanding the nature of these early galaxies is a forefront problem in cosmology and astrophysics. TMT and IRIS will explore this "First Light" epoch by revealing extremely faint star-forming galaxies and by studying the detailed physical characteristics of individual early galaxies.

The James Webb Space Telescope (JWST) has the sensitivity and spatial resolution designed to find early $(z>7)$ galaxies using its broad-band imaging capabilities. The capabilities of TMT/IRIS will be extremely complementary, allowing the discovery of line-emitting (Lyman $\alpha$ ) populations that are, most likely, distinct 


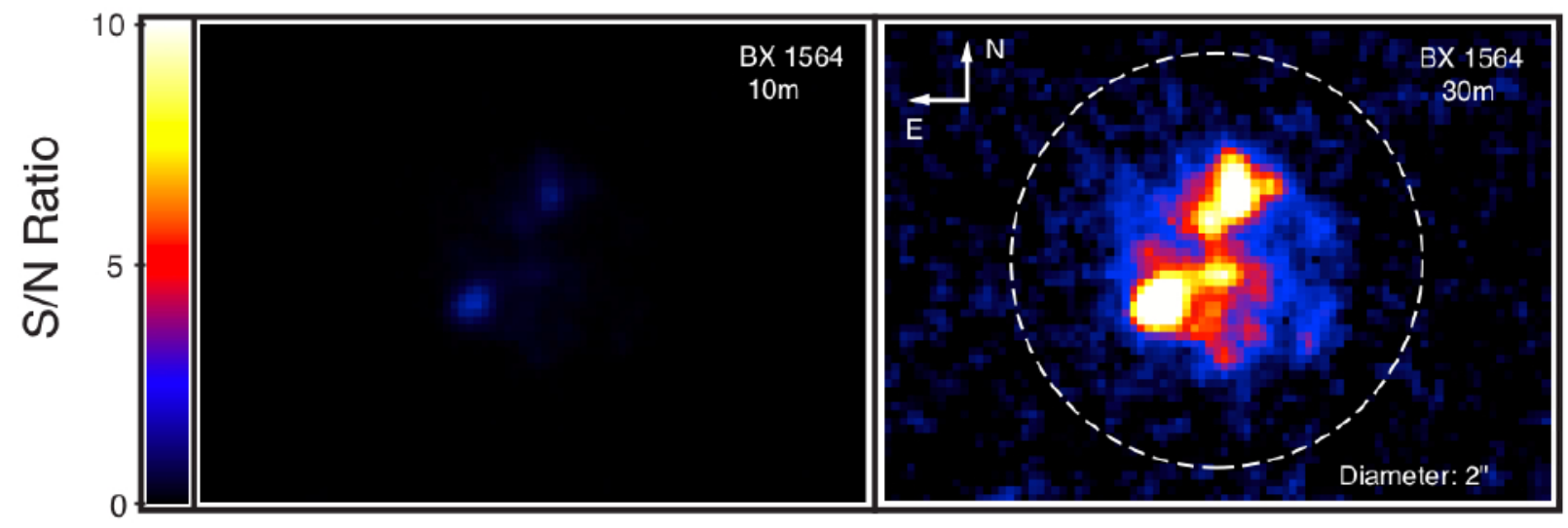

Figure 4. Relative signal-to-noise ratios expected for a two-hour observation of $\mathrm{H} \alpha$ emission from the star-forming galaxy HDF-BX1564 with current 10m (left-hand panel) and next-generation 30m (right-hand panel) facilities, from. ${ }^{18}$ Note the key increase in the ability to detect low surface brightness emission on small angular scales between the two galaxy components.

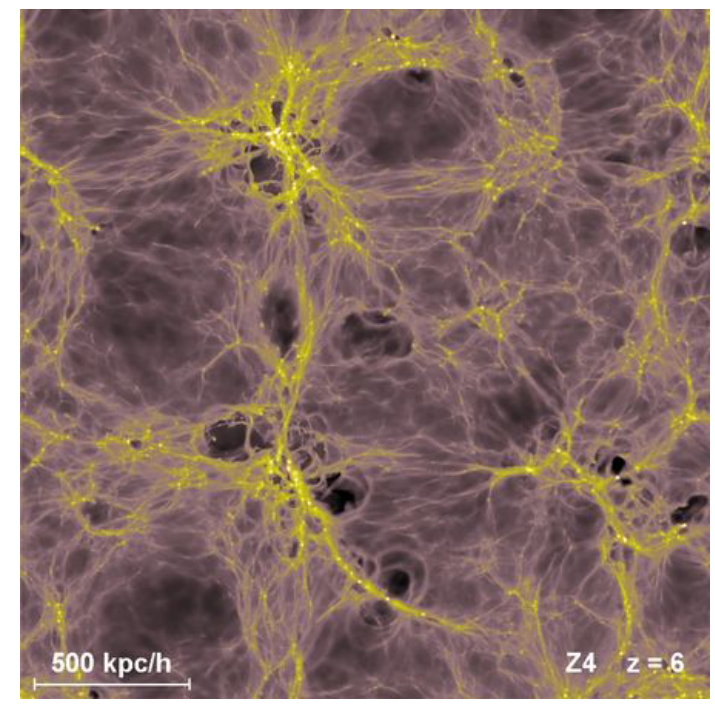

Figure 5. A hydrodynamic simulation of the early baryonic density of a $\Lambda$ CDM universe, from. ${ }^{21}$ At very early epochs, the star-forming knots in the universe may be detectable as Lyman $\alpha$ emitting sources in the observed-frame near infrared. IRIS will excel at detecting and characterizing these sources. 
from the "Lyman break" galaxy populations JWST will find. The Lyman $\alpha$ emitters may be younger and lowermass objects, potentially containing less metal-enriched and more massive stars that differ in character from star formation at the current epoch. With the most sensitive spectroscopy available in the near-infrared, IRIS will also study the physical properties of all types of star-forming first-light galaxies.

The most fundamental question about first light is the number density of star-forming galaxies at the earliest epochs; these are the key sources of ionizing photons. The combination of TMT and IRIS will contribute most to the problem of finding faint Lyman $\alpha$ emitters in the $7<z<19$ range. By targeting regions around large galaxies discovered by JWST or the gravitationally lensed regions around known nearby massive galaxy clusters, we can explore different regions of the Lyman $\alpha$ luminosity function.

The epoch of the reionization of the universe likely began around the most rapidly star-forming galaxies and proceeded outward in "bubbles" that eventually grew together to fill space. Because Lyman $\alpha$ emission does not penetrate a neutral medium, the clustering of Lyman $\alpha$ sources on Mpc scales actually reveals the growth of these bubbles throughout cosmic time. Thus, with narrow-band maps of large areas of sky, IRIS will constrain the luminosity function and the clustering of Lyman $\alpha$ sources and reveal extensive information about the nature of reionization.

A very early epoch of reionization of the universe, as indicated by the results of the WMAP satellite, ${ }^{22,23}$ requires star formation that is very efficient at emitting ionizing photons. In addition, models of the formation of stars in pristine, metal-free gas suggest that the earliest stars were very massive, and thus had extremely hard radiation fields. This "Population III" star formation is widely sought as evidence that our theories of the early epochs in the universe are correct. Currently, the best prospect for direct detection of Population III star formation is spectroscopic evidence from the rest-frame ultraviolet, which has been redshifted to the near infrared. Deep IRIS spectroscopy of pre-selected targets will allow detections of even faint HeII lines from Population III stars. Spectroscopic observations of the profile shape of the Lyman $\alpha$ line will also reveal the characteristics of the local intergalactic medium. Taken together, the "first light" science case drives requirements on the sensitivity of IRIS, as well as the need for high enough spatial resolution to reach between night sky lines and to resolve the Lyman $\alpha$ line profiles of faint, high-redshift sources.

\section{ACKNOWLEDGMENTS}

The authors gratefully acknowledge the support of the TMT partner institutions. They are the Association of Canadian Universities for Research in Astronomy (ACURA), the California Institute of Technology and the University of California. This work was supported as well by the Gordon and Betty Moore Foundation, the Canada Foundation for Innovation, the Ontario Ministry of Research and Innovation, the National Research Council of Canada, Natural Sciences and Engineering Research Council of Canada, the British Columbia Knowledge Development Fund, the Association of Universities for Research in Astronomy (AURA), the U.S. National Science Foundation, and the National Astronomical Observatory of Japan (NAOJ).

\section{REFERENCES}

[1] Larkin, J. E., Moore, A. M., Barton, E. J., Bauman, B., Bui, K., Canfield, J., Crampton, D., Fletcher, M., Hale, D., Loop, D., Niehaus, C., Phillips, A. C., Reshetov, A., Simard, L., Smith, R., Suzuki, R., Usuda, T., and Wright, S. A., "The Infrared Imaging Spectrograph (IRIS) for TMT: Instrument Overview," in [Society of Photo-Optical Instrumentation Engineers (SPIE) Conference Series], Society of Photo-Optical Instrumentation Engineers (SPIE) Conference Series 7735 (July 2010).

[2] Wright, S. A., Barton, E. J., Larkin, J. E., Moore, A. M., Crampton, D., and Simard, L., "The Infrared Imaging Spectrograph (IRIS) for TMT: Sensitivities and Simulations," in [Society of Photo-Optical Instrumentation Engineers (SPIE) Conference Series], Society of Photo-Optical Instrumentation Engineers (SPIE) Conference Series $\mathbf{7 7 3 5}$ (July 2010).

[3] Herriot, G., Andersoen, D., Atwood, J., Boyer, C., Byrnes, P., Conan, R., Ellerbroek, B., Fitzsimmons, J. T., Gilles, L., Hickson, P., Hill, A., Jackson, K. J., Lardiére, O., Pfrommer, T., Véran, J., Wang, L., and Wevers, I., "NFIRAOS: facility adaptive optics system for the TMT," in [Society of Photo-Optical Instrumentation Engineers (SPIE) Conference Series], Society of Photo-Optical Instrumentation Engineers (SPIE) Conference Series $\mathbf{7 7 3 6}$ (July 2010). 
[4] Macintosh, B., Troy, M., Doyon, R., Graham, J., Baker, K., Bauman, B., Marois, C., Palmer, D., Phillion, D., Poyneer, L., Crossfield, I., Dumont, P., Levine, B. M., Shao, M., Serabyn, G., Shelton, C., Vasisht, G., Wallace, J. K., Lavigne, J., Valee, P., Rowlands, N., Tam, K., and Hackett, D., "Extreme adaptive optics for the Thirty Meter Telescope," in [Society of Photo-Optical Instrumentation Engineers (SPIE) Conference Series], Society of Photo-Optical Instrumentation Engineers (SPIE) Conference Series 6272 (July 2006).

[5] Marois, C., Macintosh, B., Barman, T., Zuckerman, B., Song, I., Patience, J., Lafrenière, D., and Doyon, R., "Direct Imaging of Multiple Planets Orbiting the Star HR 8799," Science 322, 1348- (Nov. 2008).

[6] Macintosh, B., Graham, J., Palmer, D., Doyon, R., Gavel, D., Larkin, J., Oppenheimer, B., Saddlemyer, L., Wallace, J. K., Bauman, B., Evans, J., Erikson, D., Morzinski, K., Phillion, D., Poyneer, L., Sivaramakrishnan, A., Soummer, R., Thibault, S., and Veran, J., "The Gemini Planet Imager," in [Society of Photo-Optical Instrumentation Engineers (SPIE) Conference Series], Society of Photo-Optical Instrumentation Engineers (SPIE) Conference Series 6272 (July 2006).

[7] Weinberg, N. N., Milosavljević, M., and Ghez, A. M., "Stellar Dynamics at the Galactic Center with an Extremely Large Telescope," ApJ 622, 878-891 (Apr. 2005).

[8] Zucker, S., Alexander, T., Gillessen, S., Eisenhauer, F., and Genzel, R., "Probing Post-Newtonian Physics near the Galactic Black Hole with Stellar Redshift Measurements," ApJ Letters 639, L21-L24 (Mar. 2006).

[9] Olling, R. P. and Merrifield, M. R., "Two measures of the shape of the dark halo of the Milky Way," MNRAS 311, 361-369 (Jan. 2000).

[10] Barth, A., Cote, P., Milosavljevic, M., Seth, A., van der Marel, R., and Volonteri, M., "The Nuclei of Low-Mass Galaxies and the Search for the Smallest Massive Black Holes," in [astro2010: The Astronomy and Astrophysics Decadal Survey], ArXiv Astrophysics e-prints 2010, 12-+ (2009).

[11] Volonteri, M., Lodato, G., and Natarajan, P., "The evolution of massive black hole seeds," MNRAS 383, 1079-1088 (Jan. 2008).

[12] Frogel, J. A., Stephens, A., Ramírez, S., and DePoy, D. L., "An Accurate, Easy-To-Use Abundance Scale for Globular Clusters Based on 2.2 Micron Spectra of Giant Stars," AJ 122, 1896-1915 (Oct. 2001).

[13] Stephens, A. W. and Frogel, J. A., "The Structure and Stellar Content of the Central Region of M33," AJ 124, 2023-2038 (Oct. 2002).

[14] Davidge, T. J., Rigaut, F., Chun, M., Brandner, W., Potter, D., Northcott, M., and Graves, J. E., "The Peak Brightness and Spatial Distribution of Asymptotic Giant Branch Stars Near the Nucleus of M32," ApJ Letters 545, L89-L92 (Dec. 2000).

[15] Davidge, T. J., "The Brightest Asymptotic Giant Branch Stars in the Inner Bulge of M31," AJ 122, 1386-1396 (Sept. 2001).

[16] Davidge, T. J., Beck, T. L., and McGregor, P. J., "Identifying Bright Stars in Crowded Environments Using Velocity Dispersion Measurements, and an Application to the Center of M32," PASP 122, 241-247 (Feb. 2010).

[17] Law, D. R., Steidel, C. C., Erb, D. K., Larkin, J. E., Pettini, M., Shapley, A. E., and Wright, S. A., "The Kiloparsec-scale Kinematics of High-redshift Star-forming Galaxies," ApJ 697, 2057-2082 (June 2009).

[18] Law, D. R., Wright, S. A., Ellis, R. S., Erb, D. K., Nesvadba, N., Steidel, C. C., and Swinbank, M., "Kinematics and Formation Mechanisms of High-Redshift Galaxies," in [astro2010: The Astronomy and Astrophysics Decadal Survey], ArXiv Astrophysics e-prints 2010, 172-+ (2009).

[19] Wright, S. A., Law, D. R., Ellis, R. S., Erb, D. K., Larkin, J. E., Lu, J. R., and Steidel, C. C., "Tracing the Evolution and Distribution of Metallicity in the Early Universe," in [astro2010: The Astronomy and Astrophysics Decadal Survey], ArXiv Astrophysics e-prints 2010, 327-+ (2009).

[20] Wright, S. A., Larkin, J. E., Graham, J. R., and Ma, C., "The Presence of Weak Active Galactic Nuclei in High Redshift Star-forming Galaxies," ApJ 711, 1291-1296 (Mar. 2010).

[21] Springel, V. and Hernquist, L., "The history of star formation in a $\Lambda$ cold dark matter universe," $M N$ RAS 339, 312-334 (Feb. 2003).

[22] Spergel, D. N., Bean, R., Doré, O., Nolta, M. R., Bennett, C. L., Dunkley, J., Hinshaw, G., Jarosik, N., Komatsu, E., Page, L., Peiris, H. V., Verde, L., Halpern, M., Hill, R. S., Kogut, A., Limon, M., Meyer, S. S., Odegard, N., Tucker, G. S., Weiland, J. L., Wollack, E., and Wright, E. L., "Three-Year Wilkinson Microwave Anisotropy Probe (WMAP) Observations: Implications for Cosmology," ApJs 170, 377-408 (June 2007). 
[23] Dunkley, J., Spergel, D. N., Komatsu, E., Hinshaw, G., Larson, D., Nolta, M. R., Odegard, N., Page, L., Bennett, C. L., Gold, B., Hill, R. S., Jarosik, N., Weiland, J. L., Halpern, M., Kogut, A., Limon, M., Meyer, S. S., Tucker, G. S., Wollack, E., and Wright, E. L., "Five-Year Wilkinson Microwave Anisotropy Probe (WMAP) Observations: Bayesian Estimation of Cosmic Microwave Background Polarization Maps," ApJ 701, 1804-1813 (Aug. 2009). 